\title{
Antisocial behaviour in Malaysian adolescents: assessing measurement equivalence across gender differences
}

\begin{abstract}
It is unclear whether group differences reported in literature emerged based on true differences or due to measurement bias across gender. The present study aimed to assess the measurement equivalence of three constructs (physical aggression, social aggression, and rule breaking) derived from Subtypes of Antisocial Behaviour (STAB) measure across gender. A sample of 1191 school-going adolescents (41.80 \% males and $57.70 \%$ females) was used to test seven types of equivalence: configural, metric, scalar, latent variances, latent mean, latent covariances, and functional equivalence. Physical aggression demonstrated configural invariance, invariance of latent variance, invariance of latent covariance, and functional equivalence, but not metric and scalar invariances. Social aggression and rule-breaking constructs demonstrated equivalence at all levels. Females scored lower on physical aggression, social aggression, and rule breaking compared to males, indicating true gender differences in antisocial behaviour. Assessments of measurement equivalence should be conducted before drawing substantive inferences about gender differences in antisocial behaviour among adolescents.
\end{abstract}

Keyword: Gender differences; Antisocial behaviour; Measurement equivalence; Mean differences 